\title{
Markus Tünte*
}

\section{Der Funktionswandel von externen Arbeitsmärkten und die Heterogenität von Prekarisierungsrisiken**}

\section{Zusammenfassung}

Folgt man gängigen Arbeitsmarkttheorien, so unterscheiden sich interne und externe Arbeitsmärkte hinsichtlich ihrer strukturierenden Merkmale deutlich voneinander. Es besteht nach wie vor Konsens darüber, dass die Arbeitsmarktsegmente in Bezug auf die Beschäftigungsstabilität, die Integration von Arbeitskräften in betriebliche Wertschöpfungsprozesse sowie hinsichtlich der Beschäftigungsrisiken unterschiedlich strukturiert sind. Auf der Basis von qualitativen Intensivfallstudien in unterschiedlichen Segmenten der Verlagsindustrie und der Analyse der betrieblichen Nutzungsformen von SoloSelbstständigkeit wird gezeigt, dass die konzeptionell scharfe Trennung von internen und externen Arbeitsmärkten nicht aufrechtzuerhalten ist. So wird externe Beschäftigung zum Teil sehr arbeitnehmerähnlich und langfristig in allen betrieblichen Kernbereichen genutzt. Die heterogene Integration in betriebliche Wertschöpfungsprozesse führt zu neuen spezifischen Markt- und Prekarisierungsrisiken für Solo-Selbstständige. Die Befunde deuten dabei auf einen generellen Funktionswandel von externen Arbeitsmärkten hin.

\section{Change of external labour markets and new risks of precariousness}

\begin{abstract}
Internal and external labour markets are conceptualized as clearly separated in labour market theory. There is a consensus that labour market segments differ with regard to employment stability, the integration of workers into core areas of value creation, and their risks of precariousness. Based on qualitative case studies in different segments of the publishing industry and the analysis of how companies use freelance work, the results call into question the sharp distinction between internal and external labour markets. There is a long-term use of external workers in core activities of organizations, which

* Markus Tünte, Wissenschaftlicher Mitarbeiter an der Universität Duisburg-Essen, Institut für Soziologie, Lotharstr. 63, D-47048 Duisburg. E-mail: markus.tuente@uni-due.de.

** Artikel eingegangen: 15.2.2016

revidierte Fassung akzeptiert nach doppelt-blindem Begutachtungsverfahren: 26.9.2016.
\end{abstract}


leads to new specific risks of precariousness for freelancers. The results point to a change of how external labour markets are used and offer functional as well as numerical flexibility.

Key words: freelance work, segmented labour markets, precarious employment, publishing industry (JEL: J21, J42, J83, L82)

\section{Einleitung}

Die starke Ausweitung von atypischen Beschäftigungsverhältnissen hat zu einer kontroversen Debatte darüber geführt, ob die Beschäftigung im Kern von internen Arbeitsmarktsegmenten instabiler wird (vgl. zur Debatte Hauff \& Kirchner, 2013; Tünte, Apitzsch, \& Shire, 2011). So zeigen Segmentationsstudien, dass die Beschäftigungsstabilität in betriebsinternen Arbeitsmärkten auch in den wissensintensiven Tätigkeitsbereichen zurückgeht und externe Beschäftigung aufgrund zunehmender Betriebswechsel weiter an Bedeutung gewinnt (Struck, Grotheer, Schröder \& Köhler, 2007). Als Grund für die zunehmende Instabilität interner Arbeitsmärkte wird angeführt, dass auch betriebsspezifisch erworbene Kompetenzen und Qualifikationen zwischenbetriebliche Mobilität ermöglichen. Dabei wird in Frage gestellt, ob hochqualifizierte Tätigkeiten per se dauerhafte Kooperationen bzw. langfristige Beschäftigungsverhältnisse voraussetzen (Struck, 2006; Struck et al., 2007).

Auch wenn sich die Diagnosen zur Beschäftigungsstabilität unterscheiden, so ist es in der Arbeitsmarktforschung üblich, dass interne und externe Arbeitsmärkte konzeptionell deutlich voneinander getrennt werden. Im Gegensatz zu betriebsinternen Arbeitsmärkten ist die Beschäftigung in externen Segmenten besonders marktförmig und deutlich instabiler. Zudem wird die externe Personalflexibilisierung von Betrieben vor allem als numerische Anpassungsstrategie genutzt (vgl. Krause \& Köhler, 2012). Die betriebliche Nutzung dieser Art von Personalflexibilisierung sieht dabei nicht den Einsatz in betrieblichen Kernbereichen vor. ${ }^{1}$ Die Folge ist die Etablierung von Stamm- und Randbelegschaften, die sich in Bezug auf die Arbeits- und Beschäftigungsbedingungen grundlegend voneinander unterscheiden (Dütsch \& Struck, 2011; vgl. Keller \& Seifert, 2013, S. 100). Damit geraten vor allem die Markt- und Prekarisierungsrisiken von externen Arbeitskräften und die vertikale Strukturierung von Arbeitsmarktsegmenten in den Blick.

In dem vorliegenden Beitrag wird Bezug auf die Debatte zur betrieblichen Personalflexibilisierung genommen, wobei jedoch deutlich andere Schlussfolgerungen gezogen werden. Hierbei wird argumentiert, dass die Art und Weise, wie marktförmige Beschäftigung von Betrieben genutzt wird, eine trennscharfe Unterscheidung von internen und externen Arbeitsmärkten, numerischer und funktionaler Personalflexibilisierung sowie zwischen Kern- und Randbelegschaften nicht (mehr) zulässt. So bleibt die Heterogenität, was sowohl die Integration von externen Beschäftigungsformen als auch die Stabilität von Beschäftigung in externen Arbeitsmärkten betrifft, deutlich unterbelichtet. Je nach Art der Integration in betriebliche Wertschöpfungsprozesse sind externe Arbeitskräfte mit unterschiedlichen

1 In Anlehnung an Hertwig, Kirsch \& Wirth (2015, S. 34) können betriebliche Kernbereiche über den Betriebszweck definiert werden. Für eine Übersicht zu unterschiedlichen betrieblichen (Personal-)Flexibilisierungsstrategien, (vgl. Keller \& Seifert, 2007, S. 16). 
Prekarisierungs- und Marktrisiken konfrontiert. Im Folgenden werden zunächst die konzeptionellen Basisannahmen von Segmentationsansätzen zur Strukturierung von internen und externen Arbeitsmärkten skizziert (vgl. 2.1 und 2.1.1) und die Bezugspunkte zu betrieblicher Personalflexibilisierung und prekärer Beschäftigung diskutiert (vgl. 2.2 und 2.3). Auf der Grundlage von qualitativen Intensivfallstudien in der Verlagsindustrie (zur Methodik vgl. 3) wird im Anschluss die betriebliche Nutzung von externer Beschäftigung, mit Fokus auf den Einsatz von Solo-Selbstständigen, analysiert (vgl. 4). ${ }^{2}$ In kritischer Anlehnung an neuere Segmentationsansätze stehen dabei drei Fragen im Mittelpunkt dieser Analyse: Wie ist die externe Beschäftigung in betriebliche Wertschöpfungsprozesse integriert? Bestätigen oder widerlegen die Nutzungsgründe und konkreten Nutzungskontexte von externer Beschäftigung die Kern- und Randbelegschaftsthese? Etablieren sich langfristige Bindungen von externen Beschäftigten zu den Unternehmen? Dies würde auf Schließungsprozesse in externen Arbeitsmärkten hindeuten.

In dem darauffolgenden Schritt werden die Integration dieser Arbeitskräfte in betriebliche Wertschöpfungsprozesse und die Stabilität ihrer Bindungen zu den Unternehmen im Zusammenhang mit einer möglichen Prekarisierung der Erwerbslagen von Solo-Selbstständigen diskutiert. Zum Abschluss dieses Beitrags wird die Übertragbarkeit der Befunde zur Verlagswirtschaft auf andere wissensintensive Branchen sowie das verarbeitende Gewerbe thematisiert (vgl. 5). Die Zusammenschau der Studien zeigt dabei nicht nur eine Ausdifferenzierung der Nutzungsstrategien von Formen der externen Personalflexibilisierung, sondern deutet vielmehr auf einen generellen Funktionswandel von externen Arbeitsmärkten hin.

\section{Segmentationstheoretische Perspektiven auf die betriebliche Personalflexibilisierung}

Segmentationskonzepte sind nach wie vor der Bezugspunkt einer Vielzahl von Arbeitsmarktstudien (Dütsch \& Struck, 2014; Eichhorst \& Tobsch, 2015; Giesecke \& Groß, 2012; Krause \& Köhler, 2015). Zentrale Basisannahme ist dabei die Unterscheidung von internen und externen Arbeitsmärkten, wobei sich die Arbeitsmarktsegmente im Hinblick auf ihre Beschäftigungsstabilität grundlegend voneinander unterscheiden. ${ }^{3}$ So sind die Betriebszugehörigkeiten ausschließlich in internen Arbeitsmärkten langfristig und stabil. Zudem sind interne und externe Arbeitsmärkte vertikal gegliedert, d.h. jeweils in ein primäres, mit ,guten“, und ein ,sekundäres “ Segment mit „schlechten“ Erwerbspositionen strukturiert (vgl. Krause \& Köhler, 2011). Bezogen auf den deutschen Arbeitsmarkt ist üblicherweise die Rede von einem dreigeteilten Arbeitsmarkt (vgl. Sengenberger, 1987), wobei neben einem

2 Unter Solo-Selbstständigkeit wird eine (atypische) Erwerbsform verstanden, in der Selbstständige ohne eigene Beschäftigte in der Regel nicht weisungsgebunden und im Rahmen eines Auftragsverhältnisses mit Unternehmen bzw. Auftraggebern kooperieren. Abweichend hiervon gibt es Regelungen zu ,arbeitnehmerähnlichen Personen“, die in diesem Beitrag weiter unten thematisiert werden (vgl. 4).

3 Die Beschäftigungsstabilität wird in quantitativen Segmentationsstudien in der Regel über die betriebliche Beschäftigungsdauer, d.h. über ,,abgeschlossene Episoden betrieblicher Verweildauern“ (Struck et al., 2007, S. 168) bestimmt. 
internen und einem extern-berufsfachlichen auch der „Jedermanns-Arbeitsmarkt“ unterschieden wird. Vor allem im letztgenannten Arbeitsmarktsegment gilt die Erwerbsarbeit häufig als prekär, da Niedriglöhne und instabile Beschäftigung hier weit verbreitet sind.

\subsection{Die Grundannahmen neuerer Segmentationsansätze}

Aktuelle segmentationstheoretische Konzepte, die sich mit den „betrieblichen Beschäftigungssystemen“ befassen (vgl. für einen Überblick, Alewell \& Struck, 2012; vgl. Köhler, Loudovici, Struck, 2007) schließen in kritischer Anlehnung an die klassischen Segmentationskonzepte an. Hierbei werden heterogenere Strategien betrieblicher Personalpolitik berücksichtigt. Die Grundannahme ist, dass Unternehmen in unterschiedlichen innerbetrieblichen Arbeitsbereichen und Teilarbeitsmärkten auf vielfältige Weise interne und externe Formen der Personalanpassung miteinander kombinieren (Krause \& Köhler, 2012, S. 13). Analog zum internen Segment ist die Rede von „geschlossenen“ und mit Bezug auf externe Arbeitsmärkte von „offenen“ Beschäftigungssystemen. Neben der angesprochenen Dreiteilung des Arbeitsmarktes wird mit dem „offenen-tätigkeitsbasierten Beschäftigungssystem“ ein weiteres überbetriebliches Arbeitsmarktsegment unterschieden. Merkmal dieses Segments ist, dass die Tätigkeiten zumeist eine hohe Eingangsqualifikation voraussetzen, diese für die konkrete betriebliche Nutzung aber recht unspezifisch bzw. nicht passgenau ist. Die für den Arbeitsprozess erforderlichen Kompetenzen und Qualifikationen werden im Rahmen von innerbetrieblichen, aber vor allem auch überbetrieblichen Tätigkeitswechseln erworben und weiter ausgebaut (Struck, 2006).

Abb. 1: Die Strukturierung unterschiedlicher Arbeitsmarktsegmente

\begin{tabular}{|c|c|c|c|c|}
\hline & Segment & $\begin{array}{l}\text { Betriebliche } \\
\text { Beschäftigungs- } \\
\text { dauer }\end{array}$ & Qualifikation & $\begin{array}{l}\text { Einkommens- } \\
\text { differenzierung }\end{array}$ \\
\hline $\begin{array}{l}\text { primär- } \\
\text { intern }\end{array}$ & $\begin{array}{l}\text { interne Arbeitsmärkte } \\
\text { (geschlossene betriebliche Be- } \\
\text { schäftigungssysteme) }\end{array}$ & langfristig & $\begin{array}{l}\text { betriebsspezifische } \\
\text { Qualifikationen }\end{array}$ & ,gutes' Einkommen \\
\hline $\begin{array}{l}\text { sekundär- } \\
\text { intern }\end{array}$ & $\begin{array}{l}\text { interne Arbeitsmärkte } \\
\text { (geschlossene betriebliche } \\
\text { Beschäftigungssysteme) }\end{array}$ & langfristig & $\begin{array}{l}\text { betriebsspezifische } \\
\text { (einfache) Qualifikationen }\end{array}$ & Niedriglohn \\
\hline \multirow[t]{2}{*}{$\begin{array}{l}\text { primär- } \\
\text { extern }\end{array}$} & $\begin{array}{l}\text { externe Arbeitsmärkte } \\
\text { (offene betriebliche Beschäfti- } \\
\text { gungssysteme) } \\
\text { (-) berufsfachlich }\end{array}$ & $\begin{array}{l}\text { kurz- bis } \\
\text { mittelfristig }\end{array}$ & $\begin{array}{l}\text { berufsfachliche } \\
\text { Qualifikationen }\end{array}$ & gutes' Einkommen \\
\hline & (-) tätigkeitsbasiert & $\begin{array}{l}\text { kurz- bis } \\
\text { mittelfristig }\end{array}$ & $\begin{array}{l}\text { hohe (unspezifische) Ein- } \\
\text { gangsqualifikationen }\end{array}$ & ,gutes' Einkommen \\
\hline $\begin{array}{l}\text { sekundär- } \\
\text { extern }\end{array}$ & $\begin{array}{l}\text { externe Arbeitsmärkte } \\
\text { (offene unstrukturierte betrieb- } \\
\text { liche Beschäftigungssysteme) } \\
\text { (-) Jedermanns-Arbeitsmarkt }\end{array}$ & $\begin{array}{l}\text { kurz- bis } \\
\text { mittelfristig }\end{array}$ & $\begin{array}{l}\text { unspezifische, allgemeine } \\
\text { Qualifikationen }\end{array}$ & Niedriglohn \\
\hline
\end{tabular}

Quelle: Darstellung in Anlehnung an Krause \& Köhler (2012, S. 14) 
Auch in diesen Ansätzen umfassen lediglich interne Arbeitsmärkte bzw. „geschlossene betriebliche Beschäftigungssysteme" langfristige Beschäftigungsbeziehungen. Dies wird darauf zurückgeführt, dass in betriebsinternen Arbeitsmärkten Unternehmen mit Blick auf betriebliche Erfordernisse in die Qualifizierung und Weiterbildung von Beschäftigten investieren, so dass diese betriebsspezifische Qualifikationen und Wissen erwerben, was wiederum eine effizientere Verausgabung von Arbeitskraft ermöglicht. Die Leistungsbereitschaft von Beschäftigten wird dabei durch das Reziprozitätsprinzip der Leistungsverausgabung gegen Beschäftigungssicherheit gewährleistet (vgl. Dütsch \& Struck, 2013). In Bezug auf die betriebliche Nutzung von externer Beschäftigung können Bezugs- und Kontrollprobleme von Arbeitskräften wiederum durch die Standardisierung von Qualifikationen und Tätigkeitsprofilen gelöst werden (Struck \& Dütsch, 2012, S. 168f.).

\subsubsection{Die Verschränkung und Transformation von Arbeitsmarktsegmenten}

Auch wenn die Grenzen zwischen internen und externen Arbeitsmärkten in Segmentationstheorien scharf gezogen werden, können die Arbeitssegmente in Bezug auf die Personalrekrutierung stärker aneinandergebunden sein. Im Rahmen von ,extern kontrollierten Arbeitsmärkten“ (Sengenberger, 1987, S. 272f.) können Betriebe, über enge Beziehungen zu Arbeitsvermittlern oder auch über informelle Beziehungsnetzwerke, Zugriff auf den außerbetrieblichen Arbeitsmarkt nehmen, um perspektivisch Arbeitskräfte für die betriebliche Stammbelegschaft zu rekrutieren. Marsden (1999, S. 218) verweist darüber hinaus auf „hybrid cases“, die er auf die Verzahnung von internen mit extern-berufsfachlichen Arbeitsmärkte bezieht. Dies ist etwa in Betrieben der Fall, in denen Beschäftigte eine gleiche berufliche Qualifikation aufweisen, die innerbetriebliche Allokation von Arbeitskräften aber an betriebsinterne Weiterbildungs- und Aufstiegswege geknüpft ist.

In aktuellen segmentationstheoretischen Ansätzen wird zudem eine grundlegende Transformation interner Arbeitsmärkte konstatiert. So wird nicht nur konzeptionell berücksichtigt, dass interne Arbeitsmärkte ein sekundäres Segment aufweisen, in dem Beschäftigte zwar langfristig beschäftigt sind, aber einen Niedriglohn beziehen (vgl. Krause \& Köhler, 2011, S. 589; vgl. dazu auch Abb. 1), sondern es wird auch Bezug auf den sozialwissenschaftlichen Vermarktlichungsdiskurs (vgl. Sauer, 2010) genommen. Hierbei wird die Debatte zur betrieblichen Reorganisation und Internalisierung externer (Absatz-)Marktstrukturen (vgl. Mills \& Ungson, 2001) sowie zur ,indirekten Steuerung“ (Peters \& Sauer, 2005) von Belegschaften aufgegriffen. Im Zuge dieser betrieblichen Vermarktlichungsprozesse werden berufliches Handeln und beruflicher Aufstieg stärker als jemals zuvor in internen Arbeitsmärkten an horizontal strukturierte sowie leistungsbasierte Anreizsysteme gekoppelt (Knuth, 2011; Krause \& Köhler, 2011).

Allerdings bleiben „Marktgrenzenverschiebungen“ (Brinkmann, 2011, S. 46) in Richtung einer stärkeren Externalisierung von Arbeit in Form von Outsourcing oder auch der Etablierung von (Unternehmens-)Netzwerken konzeptionell unterbelichtet. So wird, vor allem in qualitativen Studien, schon seit Jahren die Ausbreitung komplexer netzwerkförmiger Kooperationsformen diskutiert, die auch eine Integration von Solo-Selbstständigen in Kernbereichen der Wertschöpfung umfassen können (vgl. zum Warenhausbereich, Wirth, 1999; für unterschiedliche Branchen vgl. Sydow \& Wirth, 1999). In jüngster Zeit rücken im Zusammenhang mit der Auslagerung von betrieblichen Produktionsbereichen sogenannte 
„Onsite“-Werkverträge in den Fokus, in deren Rahmen u.a. die Leistungserstellung per se in den Betrieben des Auftragsstellers erfolgt. Dabei sind rechtlich selbständige (Sub-)Unternehmen und deren Arbeitskräfte in Kernbereiche der betrieblichen Wertschöpfung integriert (vgl. Hertwig et al., 2015). Allerdings wird dieser „Externalisierungsdiskurs“ nicht systematisch auf segmentationstheoretische Ansätze und deren grundlegende Konzeption von internen und externen Arbeitsmärkten bezogen.

\subsection{Betriebliche Segmentierung und prekäre Beschäftigung}

Aktuelle Segmentationskonzepte weisen zweifellos klare Bezugspunkte zur Prekarisierungsforschung auf. Durch die Strukturierung von Arbeitsmärkten in unterschiedliche vertikale Segmente wird konzeptionell berücksichtigt, dass spezifische Beschäftigungsrisiken auch in internen Arbeitsmärkten auftreten, die lange Zeit per se als geschützt galten (vgl. Bosch, 2014). Dieser Befund ist wiederum anschlussfähig an die Debatte über die Expansion von prekärer Beschäftigung, in der auf das Droh- sowie Disziplinierungspotenzial von Prekarisierungsprozessen hingewiesen wird (vgl. Dörre, 2009). In der Literatur werden unterschiedliche Definitionen und Indikatoren zur Bestimmung von prekären Beschäftigungsverhältnissen diskutiert. Gemeinsamkeit dieser Definitionen ist, dass sie relational sind, d.h. einen Bezugspunkt zu etablierten gesellschaftlichen Mindest- und Normalitätsstandards haben (vgl. Motakef, 2015). In einer enger gefassten Definition werden mit Bezug auf das Normalarbeitsverhältnis Indikatoren wie die Integration in die sozialen Sicherungssysteme, das Einkommen, die Beschäftigungsstabilität sowie die Beschäftigungsfähigkeit zur Bestimmung von prekären Erwerbslagen diskutiert (Keller \& Seifert, 2013). Andere Konzepte rücken stärker die betriebliche Ebene in den Fokus. Hierbei wird unter Prekarisierung die Unterschreitung von festgelegten Standards in Betriebsvereinbarungen oder die unzureichenden Möglichkeiten der Teilhabe an der betrieblichen Mitbestimmung sowie eine mangelnde betriebliche Integration aufgrund der ,geringeren Einbindung in kollegiale Strukturen“ (Mayer-Ahuja, 2003, S. 15) verstanden.

Prinzipiell besteht Einigkeit darüber, dass nicht jede, aber eine Reihe von atypischen Beschäftigungsformen, wie etwa Leiharbeit, geringfügige und befristete Beschäftigung oder auch Solo-Selbstständigkeit, spezifischen Beschäftigungsrisiken ausgesetzt sind. Üblicherweise werden diese atypischen Beschäftigungen betrieblich als extern-numerische, d.h. zeitlich befristete Personalflexibilisierung genutzt und konzeptionell der Randbelegschaft zugeordnet (vgl. Hohendanner \& Bellmann, 2007; vgl. Krause, 2013). Die Beschäftigung ist für Erwerbskräfte, die der betrieblichen Randbelegschaft angehören, instabil, unter Umständen schlecht oder nicht existenzsichernd entlohnt und die Möglichkeiten der Weiterqualifizierung sind stark eingeschränkt oder nicht gegeben. Dies kann gravierende Auswirkungen auf die nachhaltige und langfristige Beschäftigungsfähigkeit dieser Arbeitskräfte haben. Aufgrund der peripheren Integration in die betriebliche Arbeitsorganisation kann nur unzureichend oder überhaupt „kein betriebs- und berufsspezifisches Humankapital“ aufgebaut bzw. erhalten werden (Dütsch \& Struck, 2014, S. 60). Randbelegschaften droht nicht nur der Verlust ihrer beruflichen Kenntnisse und Fähigkeiten, sie sind für betriebliche Stammbeschäftigte auch so etwas wie ein Flexibilisierungs- und Risikopuffer. 


\subsection{Solo-Selbstständigkeit: Die betriebliche Nutzung und soziale Risiken}

Der betriebliche Einsatz von externer Beschäftigung und die daraus resultierenden Beschäftigungsrisiken werden nicht nur für die mittel- und niedrigqualifizierte Arbeit diskutiert. So wird in einer aktuellen Segmentationsstudie argumentiert, dass die betriebliche Nutzung von externen Arbeitskräften im Bereich der wissensintensiven Dienstleistungen auf eine ähnliche Art und Weise erfolgt (vgl. Krause, 2013). Hierbei werden Parallelen zwischen dem Einsatz von Zeitarbeit im Produktionsbereich und der betrieblichen Nutzung von SoloSelbstständigkeit für hochqualifizierte Tätigkeiten gezogen. So sind die Tätigkeitsbereiche für beide Formen der externen Personalflexibilisierung durch Standardisierungsprozesse bestimmt. Diese erfolgen allerdings auf einem deutlich komplexeren Niveau, etwa in Form der Vergabe von Aufgabenpaketen. Der Zuschnitt von klar umgrenzten Teilaufgaben ermöglicht Betrieben dabei eine effizientere Arbeitskontrolle sowie die Sicherstellung von Leistungsbereitschaft (vgl. 2). Eine umfassende Integration in betriebliche Kernbereiche ist nicht vorgesehen, so dass auch Solo-Selbstständige der Randbelegschaft zugeordnet werden (Dütsch \& Struck, 2011; Krause, 2013).

Im Vergleich zu betriebsinternen Stammbelegschaften ist zudem die Verantwortung für die Absicherung von sozialen Risiken von den Einsatzbetrieben abgekoppelt. Insbesondere Solo-Selbstständige sind mit einer Reihe von sozialen Risiken konfrontiert, die sie eigenverantwortlich bewältigen müssen. Prinzipiell sind sie nur lückenhaft in die sozialen Sicherungssysteme integriert, indem sie etwa keinen Anspruch auf staatliche Transfers im Falle von Arbeitslosigkeit und Krankheit haben. ${ }^{4}$ Zudem ist eine gesetzliche Rentenversicherungspflicht für sie nicht vorgesehen (Schulze Buschoff, 2014). Allerdings gibt es gesetzliche Sonderregelungen, die eine selektive Integration von spezifischen Gruppen von Selbstständigen in die gesetzliche Sozialversicherung durch spezielle (Teil-)Versicherungssysteme umfassen. Hierzu zählen neben berufsständischen Versorgungssystemen für die verkammerten Berufe etwa auch die Künstlersozialkasse (KSK).

Im Gegensatz zu abhängig Beschäftigten gibt es für Solo-Selbstständige zudem keine gesetzliche Lohnuntergrenze zur Vermeidung von Niedrigsteinkommen, was auch einen Einfluss darauf haben dürfte, dass die Einkommen von Selbstständigen eine sehr große Spreizung aufweisen (vgl. Manske \& Scheffelmeier, 2015, S. 67). Eine Reihe von SoloSelbstständigen verfügen dabei über keinerlei Altersvorsorge, so dass bei ihnen ein erhöhtes Altersarmutsrisiko besteht (Gerner \& Wießner, 2012). Insbesondere die SoloSelbstständigkeit changiert damit am unteren Ende eines Kontinuums, was die wohlfahrtsstaatlichen Anspruchsrechte betrifft (Apitzsch, Shire, Heinrich, Mottweiler \& Tünte, 2015; vgl. Schulze Buschoff, 2014). Aber nicht nur aufgrund der geringen sozialstaatlichen Anspruchsrechte sind die Prekarisierungsrisiken für Solo-Selbstständige besonders hoch. Neben einer eher randständigen und der lediglich kurz- bis mittelfristigen Integration von Solo-Selbstständigen in betriebliche Kooperationsstrukturen wird zudem ihre räumliche Aus-

4 Seit 2006 besteht für (Solo-)Selbstständige, die zuvor einer Arbeitnehmertätigkeit nachgingen die Möglichkeit, sich freiwillig gegen das Risiko von Arbeitslosigkeit versichern zu lassen. Diese zunächst befristete Option wurde 2011 neu geregelt und im SGB III dauerhaft verankert. Aufgrund von Beitragserhöhungen ,ist der Anreiz deutlich gesunken, ein solches Versicherungsverhältnis abzuschließen.“(Jahn \& Springer, 2013, S. 1) 
gliederung und die unzureichende Einbindung in kollegiale Betriebsstrukturen thematisiert (Candeias, 2008; Manske \& Schnell, 2010).

\section{Datengrundlage und Methode}

Auf der Basis von qualitativen (Intensiv-)Fallstudien in der Verlagsindustrie werden nachfolgend die betrieblichen Nutzungsstrategien von Solo-Selbstständigen analysiert und es wird - was in Studien zu dieser Form der Arbeitskraftnutzung bisher kaum geschehen ist ein kritischer Bezug auf die diskutierten segmentationstheoretischen Ansätze genommen. Die Verlagswirtschaft ist besonders geeignet für diese Art von Analyse, da sie ein „Paradebeispiel“ für ein „offenes Beschäftigungssystem“ mit hohen Anteilen externer Personalflexibilisierung ist (vgl. Köhler \& Krause, 2010, S. 405f.). Auch gegenwärtig ist die Nutzung von Solo-Selbstständigen die zentrale Strategie der externen Personalflexibilisierung. So sind, einer repräsentativen Studie zufolge, rund ein Viertel der Beschäftigen in den Verlagsunternehmen Solo-Selbständige (Mottweiler \& Tünte, 2013). Wie zuvor diskutiert (vgl. 2.3) wird ihre betriebliche Nutzung mit Standardisierungsprozessen in Verbindung gebracht und ist zudem zeitlich begrenzt. Der hohe Verbreitungsgrad dieser Art von externer Personalflexibilisierung in der Verlagswirtschaft kann möglicherweise jedoch auf unterschiedliche Nutzungsformen hindeuten und würde diesem Befund widersprechen. Um dies zu eruieren, wurden zunächst im Rahmen von vier betrieblichen Intensivfallstudien mehrtägige Arbeitsplatzbeobachtungen bei klein- und mittelständischen Verlagshäusern durchgeführt. ${ }^{5}$ Je nach Verlagssegment sind die Dienstleistungen und Produkte auf den lokalen, regionalen aber auch überregionalen Markt ausgerichtet. Hinsichtlich der Unternehmensgröße entspricht die Fallauswahl der üblichen Betriebsstruktur in der Branche, da in der Verlagswirtschaft ein Großteil der Unternehmen eine Belegschaftsgröße von weniger als 250 Beschäftigten hat (Tünte, Mottweiler, Hermann, Kulenović, 2011). Neben einem Einblick in die täglichen Betriebsabläufe wurde der Fokus u.a. auf den Nutzungsumfang von externen Arbeitskräften und deren Einbindung in die Leistungserstellung gerichtet. Hieran schlossen sich in einem zweiten Schritt leitfadengestützte Interviews mit Geschäftsführern, Personalverantwortlichen, verlagsinternen Beschäftigten und Solo-Selbstständigen an. Während die Interviews mit der Geschäftsführung die Strategien zur Beschäftigungsflexibilisierung und die Nutzungskontexte von externen Arbeitskräften thematisierten, wurden z.B. Fragen nach der Art der Integration in die betriebliche Arbeitsorganisation und die Stabilität von Bindungen insbesondere Solo-Selbstständigen gestellt. Mit dieser Strategie sollten sozial erwünschte Antworten von Seiten der Unternehmensführung vermieden werden, da eine langfristige Nutzung und eine Leistungserbringung der Solo-Selbstständigen in den Betrieben vor Ort unter Umständen auf eine Scheinselbstständigkeit hindeuten könnten und in diesem Fall nicht thematisiert würden. Zudem konnten diese Fragen besonders mit Verlagsmitarbeitern unterhalb der Geschäftsführungsebene vertieft werden, da sich bei manchen Beschäftigten - trotz vorheriger Arbeitsplatzbeobachtungen - erst während der Inter-

5 Zwei der Verlagshäuser des betrieblichen Fallstudiensamples haben Tochterunternehmen in weiteren Verlagssegmenten, so dass Erhebungen bei mehreren Zeitungs- und Buchverlagen, einem Zeitschriften- sowie einem Akzidenzverlag durchgeführt wurden. 
views herausstellte, dass sie Solo-Selbstständige waren. In diesem Zusammenhang ließen sich je nach Integration in betriebliche Wertschöpfungsprozesse unterschiedliche Prekarisierungsrisiken bestimmen, die in der nachfolgenden Fallstudienanalyse aus der Perspektive von Solo-Selbstständigen herausgearbeitet werden. Insbesondere mit Blick auf die Verlagsund Medienbranche kann die Erwerbssituation von hochqualifizierten Solo-Selbstständigen als eine „Prekarisierung auf hohem Niveau“ (Manske \& Schnell, 2010, S. 720) charakterisiert werden. Hierbei können künstlerisch-kreative Motivationen, ein hohes Bildungsniveau und subjektive Autonomiegewinne in spezifischer Weise mit wirtschaftlichen Armutsrisiken ineinandergreifen (ebd.). Um eine größere Generalisierbarkeit zu erreichen, wurden weitere Interviews u.a. mit Solo-Selbstständigen, die vor allem für private aber auch öffentlich-rechtliche Mediengroßunternehmen tätig waren, geführt. Insgesamt konnten 56 Interviews mit Solo-Selbstständigen, verlagsinternen Beschäftigten, Geschäftsführern und Personalverantwortlichen realisiert werden (vgl. Abbildung 2). Die Berücksichtigung unterschiedlicher Verlagssegmente in dem Fallstudiensample zielt darauf, eine mögliche Variation der Nutzungsstrategien von externen Arbeitskräften in Abhängigkeit von unterschiedlichen Absatzmärkten ,sichtbar“ zu machen. Das Interview- und Beobachtungsmaterial wurde im Anschluss codiert und nach der Methode der „qualitativen Inhaltsanalyse“ (Mayring, 2010) ausgewertet.

$A b b$. 2: Fallstudienübersicht ${ }^{6}$

\begin{tabular}{|c|c|c|}
\hline Methodik & Erhebungsform & Anzahl \\
\hline $\begin{array}{l}\text { betriebliche Inten- } \\
\text { sivfallstudien }\end{array}$ & $\begin{array}{l}\text { leitfadengestützte Inter- } \\
\text { views, mehrtägige Arbeits- } \\
\text { platzbeobachtungen }\end{array}$ & $\begin{array}{l}\text { Unterschiedliche Verlagshäuser } \\
\text { (im Zeitungs-, Buch-, Zeitschriften-, Akzidenzsegment) } \\
\text { Gesamtzahl } \boldsymbol{d} \text {. Interviews: } \mathbf{N = \mathbf { 4 3 }} \\
\text { - } \quad \text { Geschäftsführungsebene, } \mathrm{N}=5 \\
\text { - } \quad \text { sozialversicherungspflichtige Verlagsbeschäftigte } \\
\quad \text { (verlagsinterne Beschäftigte, Personalverantwortliche), N=30 } \\
\text { - } \quad \text { Solo-Selbständige, N=8 }\end{array}$ \\
\hline $\begin{array}{l}\text { Weitere } \\
\text { (Einzel-)Fallstudien }\end{array}$ & $\begin{array}{l}\text { leitfadengestützte } \\
\text { Interviews }\end{array}$ & $\begin{array}{l}\text { Gesamtzahl } \boldsymbol{d} \text {. Interviews: } \mathbf{N = 1 3} \\
-\quad \text { sozialversicherungspflichtige Beschäftigte, } N=8 \\
-\quad \text { Solo-Selbständige in unterschiedlichen Verlagssegmenten, } N=5\end{array}$ \\
\hline
\end{tabular}

In der gängigen Arbeitsmarktliteratur wird der Rückgriff auf „Solo-Selbstständige“ als zeitlich befristete Anpassungsstrategie an personelle Kapazitätsprobleme von Betrieben diskutiert. In Abgrenzung hierzu wird nachfolgend die These vertreten, dass die Nutzungsformen externer Arbeitskraftflexibilisierung deutlich stärker zu differenzieren sind, was ihre Integration in betriebliche Wertschöpfungsprozesse und die Etablierung von auftragsübergreifenden Bindungen betrifft. Konzepte wie das des „,kontrollierten externen Arbeitsmarktes“ oder auch der „hybrid cases“ (vgl. 2.1.1) greifen hier zu kurz. So wird in beiden Konzepten davon ausgegangen, dass betriebsinterne Arbeitsmärkte einen hohen Schließungsgrad ha-

6 Ein Teil der Interviews und Arbeitsplatzbeobachtungen wurde im Rahmen eines vom Bundesministerium für Bildung und Forschung sowie dem Europäischen Sozialfonds geförderten Forschungsprojektes (Laufzeit: 2009-2013) durchgeführt. 
ben und in erster Linie über „Einstiegspositionen“ durchlässig sind. Demgegenüber wird nachfolgend gezeigt, dass die externe Arbeitskraftnutzung nicht allein der Logik einer effizienten Personalrekrutierung und unter Umständen späteren sozialversicherungspflichtigen Beschäftigung und Weiterqualifizierung dieser Erwerbskräfte in betriebsinternen Arbeitsmärkten folgt. Vielmehr werden externe Arbeitskräfte langfristig in Kernbereiche der betrieblichen Wertschöpfung integriert, ohne aber dass dies in eine sozialversicherungspflichtige Normalbeschäftigung mündet. Eine qualitative Analyse ist in dieser Hinsicht besonders fruchtbar, da sie betriebliche Nutzungsstrategien von Arbeitskraft „sichtbar machen“ kann, die sich nicht allein z.B. über den vertragsrechtlichen Status von Erwerbskräften erschließen lassen. Hierbei geht es nicht darum, einzelne Einsatzformen zu quantifizieren, sondern den Blick auf Nutzungsstrategien zu richten, die in der Segmentationsforschung bisher unterbelichtet geblieben sind.

\title{
4. Betriebliche Nutzungsstrategien jenseits interner und externer Personalflexibilisierung
}

Die erste Nutzungsform umfasst die marktnahe Integration von Solo-Selbstständigen in die betrieblichen Dienstleistungsmärkte von lokalen und regionalen Zeitungsverlagen. Diese externen Beschäftigten können als „Revier-Journalisten“ bezeichnet werden. Bei lokalen und regionalen Verlagsunternehmen werden Kooperationsbeziehungen zu entsprechenden Solo-Selbstständigen etabliert, die in den Regionen vor Ort integriert und ansässig sind. Die Nutzungsstrategie zielt darauf, ein möglichst genaues Abbild von dem lokalen Geschehen zu erhalten, damit die Berichterstattung möglichst lesernah erfolgt. Quasi als verlängerter Arm der Redaktion haben sie die Kernaufgabe, vor Ort zu recherchieren, relevante Informationen zu selektieren und Nachrichtenbeiträge und Reportagen zu erstellen:

\begin{abstract}
„Mein Zuständigkeitsbereich sind die Städte X und Y und da eben zu berichten, was die Gemeinden an Besonderheiten anzubieten haben. Veranstaltungen, das Gleiche gilt für die ganzen Gremien oder eben Gruppierungen, zum Beispiel Caritasverbände und was alles da eben in den Gemeinden zu finden ist. Da bekomme ich halt die entsprechende Information, wir machen dies oder jenes, wir haben in der offenen Ganztagsgrundschule einen besonderen Kurs und ja, dann fahre ich hin, sammele Informationen, mach meine Fotos und berichte dann in der Zeitung X." (Solo-Selbstständiger, Z-RJ) $^{7}$
\end{abstract}

Ähnlich wie bei Stammbeschäftigten ist durch die lokale Einbettung der Leistungserstellung ein stark personalisierter Kontakt nicht nur zu „Informanten“, sondern auch zu potenziellen Lesern gegeben. Diese Form von Solo-Selbstständigkeit wird dabei als eine Art Marktstrategie genutzt, die auf eine Leserbindung durch eine möglichst lokal zentrierte Berichterstattung ausgerichtet ist. Zusätzlich haben diese Solo-Selbstständigen neben der inhaltlichen auch eine zeitliche Entlastungsfunktion der Zeitungsredaktion, da sie zeitlich flexibel eingesetzt werden können. Auch wenn diese „Freien“ nicht in sämtliche Bereiche der Betriebsorganisation eingebunden und eher an den „organisationalen Rändern“ tätig sind,

7 Die Kürzel nach den Interviewzitaten lassen sich wie folgt aufschlüsseln: Nach der Erwerbsform folgt das Verlagssegment, in dem die entsprechenden Solo-Selbstständigen überwiegend tätig sind ( $\mathrm{Z}=$ Zeitung, $\mathrm{BV}=$ Buchverlag, ZE= Zeitschrift) sowie die betriebliche Nutzungsform (RJ= Revierjournalist, VR= virtuelle Redakteure, FF= feste Freie). 
greift die rigide Trennung von Kern- und Randbelegschaften zur Beschreibung der betrieblichen Segmentierung zu kurz. Einerseits verschwimmen bei dieser Nutzungsform die in Segmentationstheorien übliche Trennung von numerischer und funktionaler Flexibilisierung, da Solo-Selbstständige in erster Linie nicht in standardisierten Aufgabenbereichen eingesetzt werden. Andererseits ist diese Art der Personalflexibilisierung prinzipiell nicht auf eine kurz- und mittelfristige Nutzungsdauer von bestimmten Arbeitskräften beschränkt. So verfügen diese Solo-Selbstständigen über Arbeitsprozesswissen, das stark auf einen lokal zugeschnittenen Markt bezogen ist, was wiederum zu sehr langfristigen Beschäftigungsbindungen führen kann:

„Was ich allerdings seit fünfzehn Jahren mache, ich schreibe für die X-Zeitung (...) und im Rahmen meiner Tätigkeit hier in Stadt X bin ich an einen Vorstand, nein Gemeinderat geraten, der mich fragte: Hören Sie mal, haben Sie nicht Zeit auch für die Y-Zeitung zu schreiben, die haben mal angefragt. Und über diese Anfrage bin ich dann eben bei der Y- Zeitung gelandet. Also wie gesagt, X-Zeitung, Z-Zeitung seit fünfzehn Jahren und Y-Zeitung müsste jetzt auch schon dreizehn Jahre sein.“ (Solo-Selbständiger, Z-RJ)

Zum Teil sind diese Bindungen auch vertraglich fixiert. So werden manche dieser „Freien“ als sogenannte „Pauschalisten“ beschäftigt. Entsprechende Solo-Selbstständige erhalten einen monatlichen Pauschalbetrag für einen vorher definierten und vertraglich festgelegten Arbeitsumfang. ${ }^{8}$

Demgegenüber lässt sich eine zweite Nutzungsform von Solo-Selbstständigkeit unterscheiden. Diese wird in Verlagen genutzt, in denen die Arbeitsabläufe räumlich stark dezentral organisiert sind und die Arbeitsteilung vornehmlich nicht mit Blick auf lokal zentrierte Absatzmärkte strukturiert ist. Dies gilt für die Dienstleistungserstellung bei überregionalen Zeitungen, Zeitschriften oder auch Buchverlagen, wobei diese Solo-Selbstständigen als „virtuelle Redakteure“ bezeichnet werden können. Ihre Kernaufgabe ist die themenspezialisierte Erstellung oder auch Lektorierung von Textbeiträgen und Publikationen. Sie haben damit eine klassische Zulieferfunktion von Nachrichten- und Text- sowie Bilddienstleistungen. Zudem sind diese Selbstständigen eine flexible Erweiterung der Themen- und Spezialkompetenzen von betriebsinternen Verlagsredaktionen und -lektoraten, indem sie in der Regel spezialisierte Teilaufgaben dezentral bearbeiten und gegebenenfalls auch redaktionelle Funktionen ausüben. Der Auftragsumfang der ausgelagerten Dienstleistungen kann dabei beträchtlich variieren. Auch hier weist das Kern- und Randbelegschaftstheorem Unschärfen auf. So sind die Tätigkeiten, die an externe Arbeitskräfte ausgelagert werden, nicht zwangsläufig standardisiert:

\footnotetext{
„Das umfasst alle Bereiche von Buchproduktion. Das heißt angefangen von Text über Lektorat, und aber auch die graphischen Arbeiten. Sowohl Layout-Entwürfe bis hin zum klassischen Buchsatz dann eben, also das mache ich, decke ich ab. Das heißt nicht, dass ich das für jedes Buchprojekt mache (...). Aber im Prinzip kann ich alles anbieten (...) Das Projekt was ich jetzt letzte Woche abgeschlossen habe, ist auch hier für den X-Verlag (...) und da habe ich aber eine Komplett-Produktion übernommen (...).“ (Solo-Selbstständige, BV-VR)
}

Im Extremfall wird diese Form von Selbstständigkeit sogar für die Besetzung von Chefredaktionen genutzt, wie dies etwa der Fall bei sehr anzeigenorientierten Zeitschriften ist. Auch im Zusammenhang mit der zweiten Nutzungsform von Solo-Selbstständigkeit können

8 Die Laufzeit dieser Verträge muss prinzipiell nicht befristet sein. Im Rahmen von Kündigungsfristen können diese allerdings sowohl vom Auftraggeber als auch vom Auftragnehmer aufgelöst werden. 
sich langfristige Kooperationsstrukturen etablieren. Allerdings basieren diese in der Regel weniger auf einer vertraglich festgelegten regelmäßigen Zusammenarbeit, sondern auf zeitlich unregelmäßigeren, aber wiederholten Auftragsbeziehungen. ${ }^{9}$ Begünstigt wird diese Form der Zusammenarbeit durch persönliche und informelle Bindungen zu Auftraggebern bzw. Unternehmen, die sich im Laufe der Dienstleistungserstellung etablieren. Wiederholte Kooperationen werden dadurch vereinfacht, dass Selbstständige auch bei dieser betrieblichen Nutzungsform kontextbezogenes Erfahrungswissen erlangen. Dies umfasst Wissen über die konkreten Bedürfnisse, Gepflogenheiten, Konventionen von Auftraggebern aber auch „extra-funktionale Aspekte“ (Dahrendorf, 1956) wie die Persönlichkeit und die Sympathie (Apitzsch, 2010).

Die dritte Nutzungsstrategie von Solo-Selbstständigkeit umfasst die arbeitnehmerähnliche Integration in Kernbereiche der betrieblichen Wertschöpfung. Diese Form der Arbeitskraftflexibilisierung wird sowohl im Bereich der Hörfunk- und Fernsehproduktionen als auch in der Verlagswirtschaft als ,feste freie Mitarbeit" bezeichnet (vgl. Gottschall, 1999). Die Dienstleistungserstellung dieser Solo-Selbstständigen ist nicht dezentral organisiert, da sie sowohl inhaltlich als auch räumlich in sämtliche redaktionellen Verlagsabläufe eingebunden sind, so dass sie wie interne Beschäftigte für die Generierung und Organisation von Medieninhalten zuständig sind. Im Hörfunkbereich und bei Zeitungen sind die entsprechenden „Freien“" in die Kernredaktion eingebunden, bei Buch- und Zeitschriftenverlagen koordinieren sie beispielsweise auch die gesamte Programm- und Ausgabenplanung:

„Ich habe fast das Gefühl, wir sind hier Mädchen für alles (...) Ja, ansonsten sind es die drei Bausteine Vertragswesen, kreative Entscheidungen, Programmentwicklung, ja und eigentlich Kalkulieren. Also Controlling, Programmentwicklung, wie könnten wir die und die Reihe, welche Themen könnten wir besetzen, welche Autoren, ja und dann eben halt auch das gesamte Vertragswesen machen und dann eben diese hunderttausend Kleinigkeiten (...). Die gesamte Projektkoordination, ja das habe ich ehrlich gesagt vergessen; das wäre im Prinzip die vierte Säule. Das wir natürlich auch die ganzen laufenden Projekte koordinieren. Jeder hat so seine anderen Freien (...).“ (Solo-Selbstständige, BV-FF)

Aufgrund dieser umfassenden Integration in die Arbeitsorganisation etablieren sich langfristige und arbeitnehmerähnliche Beziehungen. Im Gegensatz zu den anderen Formen von Alleinselbstständigkeit können diese Beziehungen kollektivvertraglich reguliert sein. ${ }^{10} \mathrm{Hie}-$ raus können sich arbeitnehmerähnliche Sozialrechte wie ein Mindesturlaubsanspruch, der Anspruch auf Bildungsurlaub und Einbeziehung in den betrieblichen Arbeits- und Gesundheitsschutz ergeben. Allerdings handelt es sich dabei zumeist nicht um kollektivvertraglich geschlossene Regelungen, sondern häufig um informelle Vertragsvereinbarungen auf betrieblicher Ebene. Die umfassende Integration in die betriebliche Arbeitsorganisation er-

9 In der Regel kooperieren die „virtuellen Redakteure“ auf der Basis von Werkverträgen mit den Verlagen. Bei Vertragsnehmern von Werkverträgen handelt es sich „um Selbstständige oder juristische Personen, die ein vereinbartes Arbeitsergebnis erbringen. Sie schulden dem Auftraggeber die Herbeiführung eines Erfolges, die Herstellung eines Werkes." (Seifert, Amlinger \& Keller, 2015, S. 2)

10 Dies kann etwa der Fall sein, wenn Solo-Selbstständige als ,,arbeitnehmerähnliche Personen“ eingestuft werden. Sozialrechtlich ist ein arbeitnehmerähnlicher Erwerbsstatus dann gegeben, wenn diese „Freien“ „überwiegend für eine [natürliche oder juristische, M.T.] Person tätig sind oder ihnen von einer Person im Durchschnitt mehr als die Hälfte des Entgelts zusteht, das sie für ihre Erwerbstätigkeit insgesamt erhalten.“ (Bleses, 2010, S. 129) Außerhalb des öffentlich-rechtlichen Sektors ist die „feste freie Mitarbeit“ allerdings selten kollektivvertraglich reguliert (Bleses, 2008, S. 112). 
möglicht wiederum den Zugang zu organisationsspezifischem Kontextwissen und die Verstetigung der Beschäftigungsbindung. Dies umfasst beispielsweise hochgradig implizites Wissen über betriebsinterne Produktionsabläufe, über Partnerfirmen sowie über betriebsspezifische Dienstleistungs- und Kundenmärkte.

\section{Die Betriebliche Integration von Solo-Selbstständigen und die Ambivalenz von Markt- und Prekarisierungsrisiken}

In Bezug auf die zuvor skizzierten drei Nutzungsformen wird nachfolgend die These vertreten, dass die Nutzung externer Beschäftigung nicht nur die Etablierung langfristiger Bindungen zur Folge hat, sondern auch zu unterschiedlichen Markt- und Prekarisierungsrisiken für Solo-Selbstständige führt. Diese Risiken sind nicht allein auf den sozial- und vertragsrechtlichen Status von Selbstständigen zurückzuführen, sondern lassen sich nur angemessen begreifen, wenn zudem die heterogene Integration von externen Arbeitskräften in betriebliche Wertschöpfungsprozesse angemessen berücksichtigt wird. Diese Perspektive auf prekäre Beschäftigung ist im Diskurs über die Auswirkungen der Arbeitsmarktsegmentierung und des Beschäftigungswandels auf soziale Ungleichheitslagen unterbelichtet geblieben.

Mit einem hohen materiellen Prekarisierungsrisiko sind „Revier-Journalisten“ konfrontiert, die dezentral und marktnah in lokalen Absatzmärkten tätig sind. Eine Konzentration, beispielsweise auf das lokale Zeitungssegment führt zu einem erhöhten materiellen Prekarisierungsrisiko, da die entsprechenden Honorare selten existenzsichernd sind. Dies ist häufig auch der Fall, wenn die Bindungen sehr langfristig und wie im Falle der „Pauschalisten" vertraglich reguliert sind. Es ist daher nicht verwunderlich, dass diese Form von SoloSelbstständigkeit häufiger als Zuverdienst zum Haushaltseinkommen ausgeübt wird:

\footnotetext{
„Der Nachteil ist klar, wenn ich aufs Konto gucke, der Spaßfaktor ist höher als mein Kontostand (...). Ich meine man kann ruhig über Zahlen sprechen; schlechtester Monat liegt unter zweihundert Euro. Gute Monate liegen jetzt was die X-Zeitung angeht bei 400 bis 500 Euro (...). Und deswegen bin ich sehr froh, dass ich eine Frau habe, die mein zeitintensives Hobby entsprechend finanziert.“ (Solo-Selbstständiger, Z-RJ)
}

Es gibt unterschiedliche Kompensationsstrategien, auf die Solo-Selbstständige im Umgang mit materieller Prekarisierung zurückgreifen (Betzelt, 2008). So können Niedrigeinkommen durch den Haushaltskontext etwa aufgrund eines höheres Einkommens oder des sicheren sozialrechtlichen Status eines weiteren Familienmitglieds kompensiert werden. Zudem kann diese Form von Selbstständigkeit lediglich eine transitorische Phase in der beruflichen Biographie sein. Die Diversifizierung des eigenen Dienstleistungsportfolios ist eine weitere Strategie zur Kompensation von materiellen Risiken (vgl. Betzelt, 2006; vgl. Dex, Willis, Paterson \& Sheppard, 2000). Vor allem Solo-Selbstständige, die stark dezentral in den Produktionsprozess eingebunden sind („virtuelle Redakteure“), müssen anspruchsvolle und nachhaltige Marktbehauptungsstrategien verfolgen. Hierbei geht es allerdings nicht nur darum ein existenzsicherndes oder möglichst hohes Einkommen zu erzielen, sondern auch um die Sicherstellung der eigenen Beschäftigungsfähigkeit. So müssen die arbeitsbezogenen Kontakte und die Akquise dieser „Freien“ auf verschiedene Auftraggeber und unter Umständen auf andere Verlagssegmente ausgerichtet sein, um sowohl ein ausreichendes Einkommen zu gewährleisten als auch eine schwache Auftragssituation überbrücken zu kön- 
nen. Hierbei kann der Aufwand für die Pflege von informellen Auftraggeberbeziehungen besonders hoch sein (vgl. Henninger \& Gottschall, 2005). Allerdings kann der vergleichsweise höhere Grad der Kommodifizierung von manchen Selbstständigen wiederum durch inhaltliche und zum Teil zeitliche Autonomiegewinne kompensiert werden (Dörre, 2005, S. 253; Manske \& Schnell, 2010).

Demgegenüber sind die „festen Freien“ aufgrund ihrer arbeitnehmerähnlichen Integration mit anderen Beschäftigungsrisiken konfrontiert. So kann bei dieser betrieblichen Einsatzstrategie von Solo-Selbstständigkeit ein vergleichsweise höherer Grad der DeKommodifizierung gegeben sein. In diesem Fall greifen kollektivvertragliche Regelungen für arbeitnehmerähnliche Personen, wie sie bei großen Zeitungsverlagen oder bei öffentlich-rechtlichen Fernseh- und Rundfunkanstalten getroffen werden. Diese umfassen den Zugang zu bestimmten sozialen Anspruchsrechten. Die „festen Freien“, die von diesen Regelungen nicht erfasst werden, befinden sich wiederum in einer Art „regulatorischem Schwebezustand“. Sie sind zwar arbeitnehmerähnlich in die betriebliche Arbeitsorganisation integriert, aber wie andere Selbstständige haben sie nur einen lückenhaften Sozialversicherungsschutz.

In der Regel kooperieren die „festen Freien“ mit nur sehr wenigen Auftraggebern bzw. sind von einem bestimmten Unternehmen abhängig. Dies ermöglicht ihnen zumeist ein vergleichsweise hohes Einkommen und eine hohe Auftragskontinuität. Aufgrund der umfassenden Integration in betriebliche Wertschöpfungsprozesse sind die Möglichkeiten breit gefächerte Marktbehauptungsstrategien zu etablieren, jedoch deutlich eingeschränkt.

Es besteht daher die Gefahr von „Lock-in-Effekten“, d.h. einer starken wirtschaftlichen Abhängigkeit von einem Auftraggeber, der die Kooperationsmöglichkeiten etwa zu anderen Verlagen stark einschränken kann. Besonders deutlich zeigt sich dies bei Unternehmen, die externe Arbeitskräfte als „feste Freie“ an sich binden möchten. Dies kann in deutlichem Widerspruch zu der intrinsischen Motivation stehen, möglichst dezentral und nicht permanent für ein bestimmtes Unternehmen tätig zu sein:

,... ich meine ich habe zwei, drei Jahre für die (Redaktionsbüro) gearbeitet und immer mehr Verantwortung bekommen, auch Projekte selber geleitet und so. Dann habe ich irgendwann, das kam eigentlich von mir, dass ich gesagt habe, wenn ich jetzt so weiter mache, dann habe ich irgendwann das Problem, dass ich scheinselbstständig bin. Weil ich zu viel für die arbeite und deshalb habe ich gesagt, ich würde gerne reduzieren, auch um wieder Zeit für einige Projekte zu haben. Und dann haben sie halt gesagt, sie bieten mir an mich Vollzeit zu beschäftigten, dann hatte ich mich aber dagegen entschieden und da haben sie mir deutlich weniger Aufträge gegeben." (SoloSelbstständiger, Z-VR)

Starke Abhängigkeitsverhältnisse gegenüber Auftraggebern können wiederum in eine Scheinselbstständigkeit münden. Insbesondere „feste Freie“, deren Integration nicht kollektivvertraglich reguliert ist, befinden sich in einer sozialrechtlich unzureichend regulierten Grauzone zwischen abhängiger Beschäftigung und unternehmerischer Alleinselbstständigkeit. Wie die Interviews zeigen, werden zu erbringende Leistungen und Honorare häufig nicht im Voraus vertraglich, sondern auf Basis von mündlichen Absprachen festgelegt und durch eine Rechnungsstellung nach dem Leistungsprozess abgerechnet. In Zonen ,rechtlicher Prekarität" wie dieser, gewinnen informelle Aushandlungsprozesse über Arbeitsbedingungen demnach stark an Bedeutung (vgl. Apitzsch et al., 2015; Mayer-Ahuja, 2013). Hierbei müssen diese „Solo-Selbstständigen“ eine Art Balanceakt vollbringen mit ambivalenten 
Folgen für ihre Beschäftigungsfähigkeit. Die Integration in betriebliche Kernbereiche und der Zugang zu betriebsspezifischem Kontext- und Arbeitsprozesswissen ermöglichen ihnen zwar eine kontinuierliche Auftragslage, allerdings müssen sie stets die betrieblichen (Zeit-) Anforderungen und ihre eigenen zeitlichen Kapazitäten miteinander austarieren. Hinzu kommt, dass aufgrund ihres sozialrechtlich prekären Status ihre Beschäftigungsfähigkeit fragil bleibt. Sollten Auftraggeber oder Unternehmen längerfristiger in eine ökonomische Schieflage geraten, wären sie möglicherweise die Ersten, die von einem betrieblichen Personalabbau betroffen wären.

\section{Zusammenfassung und Schlussfolgerungen}

Die Analyse hat gezeigt, dass die segmentationstheoretische Perspektive auf die betriebliche Nutzung von externen Arbeitsmärkten deutlich zu monolithisch ist. Entgegen der gängigen Annahme, dass ein Großteil von atypischen Beschäftigungsformen nahezu ausschließlich in betrieblichen Randbereichen eingesetzt wird und die Beschäftigungsdauer lediglich kurz- bis mittelfristig sei (vgl. 2.1 und 2.3), stellen die hier diskutierten Befunde die Kern- und Randbelegschaftsthese sowie die strikte Trennung von funktionaler und numerischer Personalflexibilisierung grundlegend in Frage. Es wurde gezeigt, dass in externen $\mathrm{Ar}-$ beitsmärkten nicht nur langfristige Bindungen entstehen, sondern auch eine starke Heterogenität festzustellen ist, was die Integration von Solo-Selbstständigen in betriebliche Wertschöpfungsprozesse betrifft. Die Allokation von externen Arbeitskräften in betriebliche Wertschöpfungsprozesse lässt sich dabei entlang eines Kontinuums begreifen, welches von den organisationalen Rändern bzw. von marktnahen Absatzbereichen bis hinein in betriebliche Kernbereiche reicht und damit eine arbeitnehmerähnliche Integration umfassen kann.

Die Art der betrieblichen Allokation von Arbeitskraft hat wiederum sehr unterschiedliche Auswirkungen auf die Markt- und Prekarisierungsrisiken. Dabei greifen betriebliche Integrationsformen und der sozialrechtliche Status von Solo-Selbstständigen auf eine spezifische Art und Weise ineinander. So ergeben sich etwa starke „Lock-in-Effekte“ und Risiken für die Beschäftigungsfähigkeit bei „festen Freien“, deren Status als ,arbeitnehmerähnliche Person" vertragsrechtlich nicht abgesichert ist. Wie auch bei den anderen Nutzungsformen, die vertraglich nicht ,,arbeitnehmerähnlich“ reguliert sind, besteht nur eine lückenhafte Integration in die sozialen Sicherungssysteme, die allenfalls über eine Mitgliedschaft in der Künstlersozialkasse oder über zusätzlich freiwillig abgeschlossene (und teure) Versicherungsmitgliedschaften teilweise kompensiert werden können. Die Markt- und Prekarisierungsrisiken, die sich durch die diskutierten Nutzungsformen und die spezifische Art der Integration in die betrieblichen Wertschöpfungsprozesse ergeben, lassen sich zusammenfassend wie folgt illustrieren: 


\begin{tabular}{|c|c|c|c|}
\hline Merkmale & „Revierjournalist” & „virtuelle Redakteure” & „feste Freie” \\
\hline $\begin{array}{l}\text { Branchenseg- } \\
\text { ment }\end{array}$ & $\begin{array}{l}\text { lokale, regionale } \\
\text { Zeitungsverlage }\end{array}$ & $\begin{array}{l}\text { Buch,- Zeitschriften-, Zeitungs-, Ak- } \\
\text { zidenzverlage }\end{array}$ & $\begin{array}{l}\text { Buch-, Zeitschriften, Akzidenz- } \\
\text { verlage }\end{array}$ \\
\hline $\begin{array}{l}\text { Dauer der } \\
\text { Bindungen }\end{array}$ & $\begin{array}{l}\text { langfristige/ wiederholte } \\
\text { (Auftrags-)Beziehungen }\end{array}$ & $\begin{array}{l}\text { langfristige/ wiederholte } \\
\text { (Auftrags-)Beziehungen }\end{array}$ & $\begin{array}{l}\text { langfristige/ wiederholte } \\
\text { (Auftrags-)Beziehungen }\end{array}$ \\
\hline $\begin{array}{l}\text { Art der } \\
\text { betrieblichen In- } \\
\text { tegration }\end{array}$ & $\begin{array}{l}\text { "organisationale Ränder", } \\
\text { aber nicht zwangsläufig } \\
\text { Randbereich der Wertschöp- } \\
\text { fung }\end{array}$ & $\begin{array}{l}\text { dezentrale Leistungserstellung in } \\
\text { Kernbereichen der Wertschöpfung }\end{array}$ & $\begin{array}{l}\text { arbeitnehmerähnliche („,vor } \\
\text { Ort"-) Integration in sämtliche } \\
\text { Kernbereiche }\end{array}$ \\
\hline Vertragsformen & $\begin{array}{l}\text { Honorar-/ } \\
\text { „Pauschlistenverträge" }\end{array}$ & Werkverträge & $\begin{array}{l}\text { Werkverträge/ } \\
\text { „Rechnungsstellung“ }\end{array}$ \\
\hline $\begin{array}{l}\text { Zentrale Markt-/ } \\
\text { Prekarisierungs- } \\
\text { risiken }\end{array}$ & $\begin{array}{l}\text { materielle Prekarisierung; } \\
\text { lückenhafter Sozialversiche- } \\
\text { rungsschutz }\end{array}$ & $\begin{array}{l}\text { hoher Grad der Kommodifizierung: } \\
\text { Bindungen stark von informellen } \\
\text { (Auftrags-)Beziehungen abhängig; } \\
\text { lückenhafter Sozialversicherungs- } \\
\text { schutz }\end{array}$ & $\begin{array}{l}\text { eingeschränkte Beschäfti- } \\
\text { gungsfähigkeit, Scheinselbst- } \\
\text { ständigkeit, lückenhafter } \\
\text { Sozialversicherungsschutz }\end{array}$ \\
\hline
\end{tabular}

Abb. 3: Nutzungsformen sowie Markt- und Prekarisierungsrisiken von Solo-Selbstständigen

Es sind informelle Aushandlungsprozesse, die bei unzureichender formalrechtlicher Regulierung Einfluss auf die Arbeitsbedingungen wie die Höhe von Honoraren oder die Kontinuität von Auftragsbeziehungen von externen Arbeitskräften haben. Dies ist auch bei SoloSelbstständigen der Fall, die dezentral mit Unternehmen und Auftraggebern kooperieren. Hier ist die Etablierung auftragsübergreifender Beziehungen maßgeblich von persönlichen und informellen Bindungen abhängig, die vor allem „extra-funktionale Kompetenzen“ aber auch Arbeitsprozesswissen über unternehmensspezifische Abläufe und Konventionen umfassen (vgl. auch Apitzsch, 2010). Dabei rahmen netzwerkförmige Kooperationen zeitlich befristete Auftragsbeziehungen und führen zu einer Stabilisierung von Bindungen, indem Regeln und Routinen für eine weitere Zusammenarbeit ausgebildet werden (vgl. für die Fernsehindustrie Windeler \& Wirth, 2004).

In diesem Zusammenhang offenbart sich ein weiteres konzeptionelles Problem von segmentationstheoretischen Ansätzen. So werden die Relevanz von betriebsspezifischen Qualifikationen und (Arbeitsprozess-)Wissen für die Stabilität von Beschäftigungsverhältnissen lediglich für interne Arbeitsmärkte diskutiert. Wie gezeigt können sich jedoch auch in externen Arbeitsmärkten langfristige Beschäftigungsbindungen, gerade aufgrund der spezifischen Integrationsformen und des damit verbundenen Zugangs zu betriebsbezogenen Kontext- und Arbeitsprozesswissens, etablieren.

Auf der Grundlage dieser Befunde zur Arbeitskraftnutzung in der Verlagsindustrie lassen sich Parallelen zu anderen wissensintensiven Teilarbeitsmärkten ziehen. Hierbei lassen sich die Befunde unterschiedlicher Studien in Bezug auf die diskutierte Kritik an segmentationstheoretischen Konzepten re-interpretieren. So zeigen aktuelle Studien zu Honorarärzten (Wilkesmann, Ruiner, \& Apitzsch, 2016) sowie eine Reihe von Studien zur ITIndustrie, dass Solo-Selbstständige in Kernbereichen betrieblicher Wertschöpfung sowie in betriebsinternen Arbeits- bzw. Projektteams integriert sind (Kaiser, Bonss \& Rössing, 2013; 
Ruiner, Wilkens \& Küpper, 2013; Tünte et al., 2011). Für den Bereich der nicht-standardisierten Softwareentwicklung, in denen Kunden über den gesamten Prozess der Leistungserstellung eingebunden sein können, umfasst der Zugang zu betriebsspezifischem Wissen auch Kundenwissen, welches wiederum wiederholte Kooperationen mit denselben Selbstständigen begünstigt (Tünte et al., 2011). Zudem wird in weiteren Studien der informelle Charakter dieser Bindungen herausgestellt (Henninger \& Gottschall, 2005).

Auch die Leistungserbringung in der Film- und Fernsehindustrie erfolgt in stark räumlich zentrierten Arbeitsteams, in denen etwa bei freien Produktionsfirmen der Anteil von SoloSelbstständigen sehr hoch ist und sie häufig in Leitungsfunktionen tätig sind (Apitzsch, 2010). Im Gegensatz zu den öffentlich-rechtlichen Sendern sind die Arbeitsverträge von SoloSelbstständigen, die für freie Produktionen arbeiten, jedoch nicht kollektivvertraglich reguliert (Windeler \& Wirth, 2004). Die Stabilität von Kooperationsbeziehungen ist auch in dieser wissensintensiven Dienstleistungsindustrie stark von informellen Aushandlungsprozessen und netzwerkförmigen Bindungen abhängig (Apitzsch, 2010; Sydow \& Windeler, 1999).

Eine Ausdifferenzierung externer Personalflexibilisierung wird zudem für die industrielle Produktion diskutiert. In den Fokus rücken dabei vor allem unterschiedliche Nutzungsstrategien von Zeitarbeit (vgl. Promberger, 2012). Zum einen werden dabei „klassische Einsatzformen“" unterschieden, die sich auf einen begrenzten Zeitraum beziehen und die prozyklisch genutzt werden. Hiervon zu unterscheiden ist zum anderen die „strategische Nutzung“, die prinzipiell einen nicht zeitlich begrenzten Einsatz von Leiharbeitskräften vorsieht und den Einsatz in allen betrieblichen Bereichen umfassen kann. Holst (2009, S. 148) spricht daher auch von einem Funktionswandel von Leiharbeit, den er als ein Umsteuern der betrieblichen Personalpolitik von einer rein reaktiven Anpassung von Personalkapazitäten in Richtung einer strategischen Unternehmensführung beschreibt. In diesem Sinne ist diese Form der externen Personalflexibilisierung eine Art Sicherheitsnetz gegen das „Kapazitätsrisiko“ von Absatzmärkten. Die Parallelen vor allem zu „festen Freien“ in der Verlags- und Medienindustrie, deren Erwerbsstatus nicht kollektivvertraglich reguliert ist, sind unverkennbar.

Die Ausdifferenzierung der betrieblichen Personalflexibilisierung und Nutzungsstrategien von externer Beschäftigung in unterschiedlichen Teilarbeitsmärkten lassen sich damit nicht nur als eine dynamische und spannungsgeladene Koexistenz von Arbeitsmarktsegmenten (Krause \& Köhler, 2011) begreifen. Vielmehr ist von einer zunehmenden Verschränkung von internen und externen Arbeitsmarktsegmenten auszugehen, die Ausdruck eines Funktionswandels von externen Arbeitsmärkten in einer Reihe von Teilarbeitsmärkten ist. Demnach umfassen die Vermarktlichungsprozesse der betriebsinternen Arbeitsmärkte nicht nur eine auf Kennziffern basierende Form der Leistungskontrolle interner Belegschaften oder eine Internalisierung von Kundenstrukturen. Vielmehr wird marktförmige und externe Beschäftigung in Kernbereichen der Wertschöpfung zum Teil arbeitnehmerähnlich integriert, was die Abgrenzung von betriebsinternen Arbeitsmärkten deutlich brüchig werden lässt. Es zeigt sich damit, dass sich die in der Segmentationsforschung scharf gezogenen Grenzen zwischen internen und externen Arbeitsmärkten, zwischen Rand- und Kernbelegschaft, zwischen funktionaler und numerischer Flexibilität neu justieren. Die Folgen sind sehr heterogene und zum Teil auch ambivalente Auswirkungen auf die Markt- und Prekarisierungsrisiken von atypisch Beschäftigten. 


\section{Literatur}

Alewell, D., \& Struck, O. (Hrsg.). (2012). Schwerpunktheft: Betriebliche Beschäftigungsysteme. Industrielle Beziehungen, 19. München: Hampp Verlag.

Apitzsch, B. (2010). Flexibile Beschäftigung, neue Abhängigkeiten. Projektarbeitsmärkte und ihre Auswirkungen auf Lebensverläufe. Frankfurt a.M.: Campus Verlag.

Apitzsch, B., Shire, K., Heinrich, S., Mottweiler, H., \& Tünte, M. (2015). Flexibilität und Beschäftigungswandel. Weinheim: Beltz Juventa.

Betzelt, S. (2006). Flexible Wissensarbeit. Alleinverdienerinnen zwischen Privileg und Prekarität. ZeS-Arbeitspapier, Nr. 3. Bremen: Zentrum für Sozialpolitik.

Betzelt, S. (2008). Zur begrenzten Nachhaltigkeit flexibler Erwerbsmuster - Das Beispiel hoch qualifizierter Alleinselbstständiger In M. Szydlik (Hrsg.), Flexibilisierung. Folgen für Arbeit und Familie (S. 93-112). Wiesbaden: VS Verlag für Sozialwissenschaften. http://dx.doi.org/10.1007/978-3-531-90780-2_5

Bleses, P. (2008). Die Sozialintegration flexibler Erwerbsformen: Das Beispiel Alleinselbständigkeit. In G. Becke (Hrsg.), Soziale Nachhaltigkeit in flexiblen Arbeitsstrukturen. Problemfelder und arbeitspolitische Gestaltungsperspektiven (S. 107-122). Berlin: LIT Verlag.

Bleses, P. (2010). „Decent Work“ in flexiblen Erwerbsformen - auch ein deutsches Problem? In G. Becke, P. Bleses, W. Ritter, \& S. Schmidt (Hrsg.), ,Decent Work - Arbeitspolitische Gestaltungsperspektive für eine globalisierte und flexibilisierte Arbeitswelt (S. 119-136). Wiesbaden: VS Verlag für Sozialwissenschaften. http://dx.doi.org/10.1007/978-3-531-92572-1_8

Bosch, G. (2014). Neuordnung des deutschen Arbeitsmarktes. In K. Dörre, K. Jürgens, \& I. Matuschek (Hrsg.), Arbeiten in Europa: Marktfundamentalismus als Zerreißprobe (S. 91-106). Frankfurt a. M.: Campus Verlag.

Brinkmann, U. (2011). Die unsichtbare Faust des Marktes. Betriebliche Kontrolle und Koordination im Finanzmarktkapitalismus. Berlin: edition sigma. http://dx.doi.org/10.5771/9783845269054

Candeias, M. (2008). Die neuen Solo-Selbstständigen zwischen Unternehmergeist und Prekarität. Prokla. Zeitschrift für kritische Sozialwissenschaft, 150, 65-81.

Dahrendorf, R. (1956). Industrielle Fertigkeiten und soziale Schichtung. Kölner Zeitschrift für Soziologie und Sozialpsychologie, 8, 540-568.

Dex, S., Willis, J., Paterson, R., \& Sheppard, E. (2000). Freelance Workers and Contract Uncertainty: The Effects of Contractual Changes in the Television Industry. Work, employment and society, 14, 283-305. http://dx.doi.org/10.1177/09500170022118419

Dörre, K. (2005). Prekarität eine arbeitspolitische Herausforderung. WSI Mitteilungen, 58, 250-258.

Dörre, K. (2009). Prekarität im Finanzmarkt-Kapitalismus. In R. Castel \& K. Dörre (Hrsg.), Prekarität, Abstieg, Ausgrenzung. Die soziale Frage am Beginn des 21. Jahrunderts (S. 35-64). Frankfurt a.M.: Campus Verlag.

Dütsch, M., \& Struck, O. (2011). Muster interner und externer Personalanpassungsformen - Eine Analyse des IAB-Betriebspanels 2007. In D. Voss-Dahm, G. Mühge, K. Schmierl, \& O. Struck (Hrsg.), Qualifizierte Facharbeit im Spannungsfeld von Flexibilität und Stabilität (S. 249 -289). Wiesbaden: VS Verlag für Sozialwissenschaften. http://dx.doi.org/10.1007/978-3-531-92752-7_12

Dütsch, M., \& Struck, O. (2013). Geschlossene und offene Beschäftigungssyteme: Eine Analyse der Entwicklungen und Determinanten anhand beruflicher Mobilitätsprozesse In T. Haipeter, G. Mühge, K. Schmierl, \& O. Struck (Hrsg.), Berufliche Qualifikationen: Eine Analyse für offene und geschlossene Beschäftigungssysteme (S. 157-196). Wiesbaden: VS Verlag für Sozialwissenschaften. http://dx.doi.org/10.1007/978-3-658-02294-5_6

Dütsch, M., \& Struck, O. (2014). Atypische Beschäftigungen und berufliche Qualifikationsrisiken im Erwerbsverlauf. Industrielle Beziehungen, 21, 58-77. 
Eichhorst, W., \& Tobsch, V. (2015). Not so standard anymore? Employment duality in Germany. Labour Market Research, 48, 81-95. http://dx.doi.org/10.1007/s12651-015-0176-7

Gerner, H.-D., \& Wießner, F. (2012). Solo-Selbstständige. Die Förderung bewährt sich, der soziale Schutz nicht immer. IAB Kurzbericht 23/2012. Nürnberg: IAB.

Giesecke, J., \& Groß, M. (2012). Soziale Schließung und die Strukturierung externer Arbeitsmärkte. In A. Krause \& C. Köhler (Hrsg.), Arbeit als Ware. Zur Theorie flexibler Arbeitsmärkte (S. 91116). Bielefeld: Transcript Verlag. http://dx.doi.org/10.14361/transcript.9783839419847.91

Gottschall, K. (1999). Freie Mitarbeit im Journalismus. Zur Entwicklung von Erwerbsformen zwischen selbständiger und abhängiger Beschäftigung. Kölner Zeitschrift für Soziologie und Sozialpsychologie, 51, 635-654.

Hauff, S., \& Kirchner, S. (2013). Wandel der Arbeitsqualität. Arbeits- und Beschäftigungsbedingungen zwischen 1989 und 2006 in einer evaluativ-relationalen Perspektive. Zeitschrift für Soziologie, 42, 337-355.

Henninger, A., \& Gottschall, K. (2005). Freelancer in den Kultur- und Medienberufen: Freiberuflich, aber nicht frei schwebend. In N. Mayer-Ahuja \& H. Wolf (Hrsg.), Entfesselte Arbeit - neue Bindungen. Grenzen der Entgrenzung in der Medien- und Kulturindustrie (S. 153-183). Berlin: edition sigma

Hertwig, M., Kirsch, J., \& Wirth, C. (2015). Werkverträge im Betrieb. Eine empirische Untersuchung. Böckler Study 300. Düsseldorf: Hans-Böckler-Stifung.

Hohendanner, C., \& Bellmann, L. (2007). Atypische Beschäftigung und betrieblicher Flexibilisierungsbedarf. Ergebnisse des IAB-Betriebspanels. In B. Keller \& H. Seifert (Hrsg.), Atypische Beschäftigung - Flexibilisierung und soziale Risiken (S. 27-45). Berlin: edition sigma.

Holst, H. (2009). Disziplinierung durch Leiharbeit? Neue Nutzungsstrategien von Leiharbeit und ihre arbeitspolitischen Folgen. WSI-Mitteilungen, 62, 143-149.

Jahn, E., \& Springer, A. (2013). Auch Selbstständige nehmen Unterstützung in Anspruch. IABKurzbericht, 26/2013. Nürnberg: IAB.

Kaiser, S., Bonss, U., \& Rössing, I. (2013). Das Phänomen Freelancer aus organisationaler Perspektive. In S. Kaiser, E. Bamberg, R. Klatt, \& S. Schmicker (Hrsg.), Arbeits- und Beschäftigungsformen im Wandel (S. 85-114). Wiesbaden: Springer Gabler. http://dx.doi.org/10.1007/978-3-658-00331-9_4

Keller, B., \& Seifert, H. (2007). Atypische Beschäftigung - Flexibilität, soziale Sicherheit und Prekarität In B. Keller \& H. Seifert (Hrsg.), Atypische Beschäftigung - Flexibilisierung und soziale Risiken (S. 11-25). Berlin: edition sigma.

Keller, B., \& Seifert, H. (2013). Atypische Beschäftigung zwischen Prekarität und Normalität. Entwicklung, Strukturen und Bestimmungsgründe im Überblick. Berlin: edition sigma. http://dx.doi.org/10.5771/9783845268460

Knuth, M. (2011). Widersprüchliche Dynamiken im deutschen Arbeitsmarkt. WSI-Mitteilungen, 64, 580-587.

Köhler, C., \& Krause, A. (2010). Betriebliche Beschäftigungspolitik. In F. Böhle, G. G. Voß, \& G. Wachtler (Hrsg.), Handbuch Arbeitssoziologie (S. 387-412). Wiesbaden: VS Verlag für Sozialwissenschaften. http://dx.doi.org/10.1007/978-3-531-92247-8_13

Köhler, C., Loudovici, K., \& Struck, O. (2007). Generalisierung von Beschäftigungsrisiken oder anhaltende Arbeitsmarktsegmentation. Berliner Journal für Soziologie, 17, 387-406. http://dx.doi.org/10.1007/s11609-007-0031-y

Krause, A., \& Köhler, C. (2011). Von der Vorherrschaft interner Arbeitsmärkte zur dynamischen Koexistenz von Arbeitsmarktsegmenten. WSI-Mitteilung 64, S. 588-596.

Krause, A., \& Köhler, C. (2012). Was sind flexible Arbeitsmärkte und wie kann man sie erklären? Einleitung und Übersicht. In A. Krause \& C. Köhler (Hrsg.), Arbeit als Ware. Zur Theorie flexibler 
Arbeitsmärkte (S. 9-43). Bielefeld: Transcript Verlag. http://dx.doi.org/10.14361/transcript.9783839419847.9

Krause, A., \& Köhler, C. (2015). Unsicherheit als Element betrieblicher Personalpolitik. In I. Dingeldey, A. Holtrup, \& G. Warsewa (Hrsg.), Wandel der Governance der Erwerbsarbeit (S. 221-243). Wiesbaden: VS Verlag für Sozialwissenschaften. http://dx.doi.org/10.1007/978-3-65801238-0_9

Krause, I. (2013). Das Verhältnis von Stabilität und Flexibilität auf dem deutschen Arbeitsmarkt. Schließungs- und Flexibilisierungsprozesse betrieblicher Beschäftigungsstrukturen. Wiesbaden: VS Springer Verlag. http://dx.doi.org/10.1007/978-3-531-19602-2

Manske, A., \& Scheffelmeier, T. (2015). Werkverträge, Leiharbeit, Solo-Selbstständigkeit - Eine Bestandsaufnahme. WSI Diskussionspapiere, Nr. 195. Düsseldorf: Hans-Böckler-Stifung.

Manske, A., \& Schnell, C. (2010). Arbeit und Beschäftigung in der Kultur- und Kreativwirtschaft. In F. Böhle, G. G. Voß, \& G. Wachteler (Hrsg.), Handbuch Arbeitssoziologie (S. 699-728). Wiesbaden: VS Verlag für Sozialwissenschaften. http://dx.doi.org/10.1007/978-3-531-92247-8_24

Marsden, D. (1999). A Theory of Employment Systems: micro-foundations of societal diversity. Oxford: Oxford University Press. http://dx.doi.org/10.1093/0198294220.001.0001

Mayer-Ahuja, N. (2003). Wieder dienen lernen? Vom westdeutschen "Normalarbeitsverhältnis" zu prekärer Beschäftigung seit 1973. Berlin: edition sigma.

Mayer-Ahuja, N. (2013). Prekär, informell - weiblich? Zur Bedeutung von „Gender“ für die Aushöhlung arbeitspolitischer Standards. In H.-J. Burchardt, S. Peters, \& N. Weinmann (Hrsg.), Arbeit in globaler Perspektive. Facetten informeller Beschäftigung (S. 55-78). Frankfurt a.M.: Campus Verlag.

Mayring, P. (2010). Qualitative Inhaltsanalyse. Grundlagen und Techniken. 11. Aufl. .Weinheim: Beltz Juventa.

Mills, P. K., \& Ungson, G. R. (2001). Internal Market Structures. Subsitutes for Hierarchies. Journal of Service Research, 3, 252-264. http://dx.doi.org/10.1177/109467050133006

Motakef, M. (2015). Prekarisierung. Bielefeld: transcript Verlag.

Mottweiler, H., \& Tünte, M. (2013). Arbeitsmarktflexibilisierung in der Verlagsindustrie: Beschäftigungsstrategien im Spannungsfeld von Flexibilität und Stabilität. In J. Hafkesbrink \& K. Shire (Hrsg.), Flexibilität und Stabilität in der Verlags- und Medienbranche. Konzepte beidhändiger Unternehmen. (S. 25-64). Lohmar: Eul-Verlag.

Peters, K., \& Sauer, D. (2005). Indirekte Steuerung - eine neue Herrschaftsform. Zur revolutionären Qualität des gegenwärtigen Umbruchprozesses. In H. Wagner (Hrsg.), Rentier ich mich noch? Neue Steuerungskonzepte im Betrieb (S. 23-58). Hamburg: VSA Verlag.

Promberger, M. (2012). Topographie der Leiharbeit: Flexibilität und Prekarität einer atypischen Beschäftigungsform. Berlin: edition sigma. http://dx.doi.org/10.5771/9783845270074

Ruiner, C., Wilkens, U., \& Küpper, M. (2013). Patterns of organizational flexibility in knowldegeintensive firms - going beyond existing concepts. Management Revue, 24, 162-178.

Sauer, D. (2010). Vermarktlichung und Vernetzung der Unternehmens- und Betriebsorganisation. In F. Böhle, Voß, G. G., Wachteler, G. (Hrsg.), Handbuch Arbeitssoziologie (S. 545-568). Wiesbaden: VS Verlag für Sozialwissenschaften. http://dx.doi.org/10.1007/978-3-531-92247-8_18

Schulze Buschoff, K. (2014). Teilhabe atypisch Beschäftigter: Einkommen, Sozialversicherungsrechte und betriebliche Mitbestimmung. Arbeit, 23, 211-224.

Seifert, H., Amlinger, M., \& Keller, B. (2015). Selbstständige als Werkvertragsnehmer. Ausmaß, Strukturen und soziale Lage. WSI-Diskussionspapier, Nr. 201. Düsseldorf: Hans-BöcklerStiftung.

Sengenberger, W. (1987). Struktur und Funktionsweise von Arbeitsmärkten. Die Bundesrepublik Deutschland im internationalen Vergleich. Frankfurt a.M.: Campus Verlag. 
Struck, O. (2006). Flexibilität und Sicherheit. Empirische Befunde, theoretische Konzepte und institutionelle Gestaltung von Beschäftigungsstabilität. Wiesbaden: VS Verlag für Sozialwissenschaften.

Struck, O., \& Dütsch, M. (2012). Gesicherte Mobilität am Arbeitsmarkt: Zur Bedeutung berufsfachlicher Qualifikationen in geschlossenen und offenen Beschäftigungssystemen. Industrielle Beziehungen, 19, 154-186.

Struck, O., Grotheer, M., Schröder, T., \& Köhler, C. (2007). Instabile Beschäftigung. Neue Ergebnisse zu einer alten Kontroverse. Kölner Zeitschrift für Soziologie und Sozialpsychologie, 59, 294-317. http://dx.doi.org/10.1007/s11577-007-0030-1

Sydow, J., \& Windeler, A. (1999). Projektnetzwerke: Management von (mehr als) temporären Systemen. In J. Engelhard \& E. J. Sinz (Hrsg.), Kooperation im Wettbewerb. Neue Formen und Gestaltungskonzepte im Zeichen von Globalisierung und Informationstechnologie (S. 211-235). Wiesbaden: Springer Gabler. http://dx.doi.org/10.1007/978-3-322-86918-0_9

Sydow, J., \& Wirth, C. (Hrsg.). (1999). Arbeit, Personal und Mitbestimmung in Unternehmensnetzwerken. München: Hampp Verlag.

Tünte, M., Apitzsch, B., \& Shire, K. (2011). Neue Beschäftigungsstrategien jenseits von externer und interner Flexibilisierung. Berliner Journal für Soziologie, 21, 363-381. http://dx.doi.org/10.1007/s11609-011-0161-0

Tünte, M., Mottweiler, H., Hermann, N., \& Kulenović, D. (2011). Die Verlagsindustrie im Innovationsdilemma: Die Suche nach Bewältigungsstrategien in Zeiten des Umbruchs. In K. Meißner \& M. Engelien (Hrsg.), Virtual Enterprises, Communities \& Social Networks (S. 121131). Dresden: TUDpress.

Wilkesmann, M., Ruiner, C., \& Apitzsch, B. (2016). Hochqualifzierte Solo-Selbstständige im operativen Kern - Auswirkungen des Einsatzes von Honorarärtzen auf die Professionsorganisation Krankenhaus. In W. Matiaske \& A. Czaya (Hrsg.), Periphere Arbeit im Zentrum (S. 95-117). Baden-Baden: Nomos. http://dx.doi.org/10.5771/9783845273204-95

Windeler, A., \& Wirth, C. (2004). Arbeitsregulation in Projektnetzwerken. Eine strukturationstheoretische Perspektive. Industrielle Beziehungen, 11, 295-319.

Wirth, C. (1999). Unternehmensvernetzung, Externalisierung von Arbeit und industrielle Beziehungen. Die negotiation of order von ausgewählten Netzwerkbeziehungen einer Warenhausunternehmung. München: Hampp Verlag. 


\section{DuEPublico}

Duisburg-Essen Publications online
UNIVERSITÄT

DEUS'SEN R G

Offen im Denken

Ub | universitäts

Dieser Text wird über DuEPublico, dem Dokumenten- und Publikationsserver der Universität Duisburg-Essen, zur Verfügung gestellt. Die hier veröffentlichte Version der EPublikation kann von einer eventuell ebenfalls veröffentlichten Verlagsversion abweichen.

DOI: $\quad$ 10.3224/indbez.v24i1.04

URN: urn:nbn:de:hbz:464-20200717-124448-3 